\title{
Especificación de un modelo para el estudio del interculturalismo laboral ${ }^{12}$
}

\author{
Cruz García Lirios ${ }^{3}$
}

\section{RESUMEN}

Los estudios del Trabajo Social en torno a la migración se han enfocado en la gestión de los recursos y las capacidades de los sujetos, el diagnóstico de oportunidades, la promoción de la salud reproductiva, la prevención de enfermedades y la sistematización de indicadores de autonomía relativa para el desarrollo local. No obstante, la discusión no ha avanzado hacia los efectos del cambio climático sobre la calidad de vida de los migrantes, su bienestar subjetivo y percibido, así como la protección y la seguridad social, todos temas que debieran atraer el interés creciente de la disciplina. De este modo, el presente trabajo busca especificar un modelo en el sentido señalado, a fin de poder abordar la problemática. Para tal propósito, se llevó a cabo un estudio documental, retrospectivo y exploratorios con una selección de fuentes indexadas a repositorios de América Latina -Dialnet, Latindex y Redalyc-, considerando el año de publicación de 2010 a la fecha y las palabras clave. Se advierten trayectorias de relaciones de dependencia entre las variables revisadas en la literatura que permiten contrastar el modelo.

Palabras clave: Migración, multiculturalismo, interculturalismo, trabajo social, modelo.

\section{especificação de um modelo para o estudo do interculturalismo laboral}

\section{RESUMO}

Os estudos do Trabalho Social em torno da migração têm sido focados na gestão dos recursos e as capacidades dos sujeitos, o diagnóstico de oportunidades, a promoção da saúde reprodutiva, a prevenção de doenças e a sistematização de indicadores de autonomia relativa para o Desenvolvimento Local. No entanto, a discussão não tem apontado para os efeitos da mudança climática sobre a qualidade de vida dos migrantes,

1 Artículo recibido el 22/07/2017. Artículo aprobado el 31/12/2017

2 N.E: la traducción de las citas en inglés que aparecen en este artículo, fue realizada por Daniel Adrián Gómez Monroy, traductor y doctor en filología, Otawa University, Canadá. E-mail DgomeMonroy@hotmail.com

3 Mexicano, Psicólogo Social, Universidad Autónoma del Estado de México. E-mail garcial213@profesor.uaemex.mx 
seu bem-estar subjetivo e percebido, assim como a proteção e a segurança social, todos estes, temas que deveriam atrair o interesse crescente da disciplina. Deste modo, o atual trabalho busca especificar um modelo no sentido assinalado, a fim de poder tratar a problemática. Para tal propósito, foi efetuado um estudo documental, retrospectivo e exploratório com uma seleção de fontes indexadas a repositórios da América Latina Dialnet, Latindex e Redalyc-, considerando o ano de publicação de 2010 à data atual e as palavras-chave. São advertidas trajetórias de relações de dependência entre as variáveis revisadas na literatura que permitem contrastar o modelo.

Palavras-chave: Migração, Multiculturalismo, Interculturalismo, Trabalho Social, Modelo

\section{Specification of a model for the study of labor interculturalism}

\section{ABSTRACT}

Social Work studies about migration have focused on management of resources and capacities of subjects, diagnosis of opportunities, promotion of reproductive health, prevention of diseases and systematization of indicators for relative autonomy for Local Development. However, the discussion has not advanced towards the effects of climate change on migrants' quality of life, their subjective and perceived well-being, as well as protection and social security -subjects that should attract the growing interest of the discipline. This work seeks to specify a model to intent to address the problem. To that end, a retrospective and exploratory documentary study was carried out with a selection of sources indexed to repositories in Latin America -Dialnet, Latindex and Redalyc- considering the year of publication from 2010 to date and keywords. There are trajectories of dependency relationships among variables reviewed in the literature allowing us to contrast the model.

Keywords: Migration, Multiculturalism, Interculturalism, Social Work, Model

\section{Introducción}

El estudio se inscribe en la división de Ciencias Sociales y Humanidades, disciplina de Trabajo Social, área de políticas sociales y servicios comunitarios a grupos vulnerables, pero el modelo especificado invita a un abordaje multidisciplinar en el que la economía, la sociología y la psicología contribuirían en la discusión de las hipótesis propuestas. 
En el marco de los efectos del cambio climático en la salud pública, principalmente la salud ocupacional, el interculturalismo, entendido como un sistema de gestión y administración de las demandas del entorno respecto de las capacidades y los recursos disponibles, es una alternativa que, traducida en política social de protección y atención a grupos vulnerados, marginados y excluidos, obliga a la reflexión académica como determinante de las asimetrías entre sociedades de acogidas con respecto a grupos migrantes, así como entre actores civiles y políticos dedicados a la construcción de programas y estrategias de intervención social.

De este modo, la aproximación económica advierte que el interculturalismo abre la discusión en torno a los derechos laborales y ocupacionales que, en materia de salud pública, inciden en las políticas de atención y protección a grupos vulnerados, como es el caso de los migrantes (Fuentes y Ortiz, 2012). No obstante, desde la sociología del trabajo, el interculturalismo es resultado del desarrollo de capacidades y competencias laborales, que se exacerban en función de las demandas del mercado y las oportunidades de emprendimiento e innovación en los países receptores de migrantes (García, 2007).

Es así como la importación y exportación de mano de obra requerida para las laborales de alto riesgo ocupacional, generan un autoconcepto por parte de los trabajadores migrantes que explica los altos índices de accidentes y enfermedades relacionadas con el tipo de ocupación. De hecho, la calidad de vida y el bienestar subjetivo de los migrantes está en función de la calidad y el tipo de actividad laboral (Aysa y Cachon, 2013).

Sin embargo, las políticas sociales de atención y protección a los migrantes parecen transitar hacia un escenario diferente al que marca la aproximación económica en el rubro de la capacitación, la productividad y la competitividad, o bien, un sendero distinto al que sugiere la perspectiva sociológica de derechos laborales y prevención de enfermedades y de accidentes (García, 2008). Más bien las políticas de seguridad social parecen ser legitimadas por un enfoque psicológico de autocuidado y autoprotección de la sa- 
lud ocupacional, así como de la seguridad social que, en el caso de la vivienda, sería la modalidad de la autoconstrucción.

Por consiguiente, el interculturalismo como enfoque multidisciplinar de empleo, protección y autocuidado de los migrantes en los ámbitos económico, laboral y ocupacional, contribuye decididamente a la edificación de una política social, una estrategia de protección social y un programa de salud pública centrados en el diálogo entre las culturas migrantes con respecto a las culturas oriundas.

El interculturalismo, desde el enfoque psicológico, la perspectiva sociológica y la aproximación económica sugieren un diálogo entre organizaciones de la sociedad civil e instituciones estatales, pero, sobre todo, implican el establecimiento y distribución de poderes representativos de las minorías y grupos vulnerados (Berry, 2016).

El objetivo del presente trabajo es revisar la literatura relativa a la historia, teoría y conceptos de migración, con la finalidad de especificar un modelo para su estudio. A partir de un estudio documental con una selección de fuentes indexadas a repositorios líderes de América Latina -Dialnet, Latindex, Redalyc-, considerando las palabras clave de "migración", "aculturación", "multiculturalismo" e "interculturalismo", se especificaron dos trayectorias explicativas de las relaciones entre variables utilizadas en la literatura.

Tal modelo especificado permitirá el contraste de dos hipótesis alusivas al multiculturalismo o ajuste de los flujos migrantes a los usos y costumbres de una cultura dominante, y el supuesto del interculturalismo relativo al diálogo entre las minorías y mayorías a través de la conquista de estándares de calidad de vida y bienestar subjetivo, que permiten la producción de iniciativas y la representación de los flujos migrantes en las estructuras políticas del país receptor.

\section{Conceptos básicos en torno al Trabajo Social de la migración}

La historia del Trabajo Social en México ha estado marcada por las políticas sociales de solidaridad con los migrantes, como fue el 
caso de las llevadas a cabo por el presidente general Lázaro Cárdenas, actor intelectual de la creación de la Secretaria de Asistencia Pública, antecedente del surgimiento oficial del Trabajo Social (Valero, 1997).

Muy pronto, el énfasis en las necesidades sociales distinguió al Trabajo Social de otras disciplinas de los servicios públicos. En el caso de las culturas migrantes provenientes de Europa, Estados Unidos y América del Sur, éstas advertían necesidades para su subsistencia y para su desarrollo. En este rubro, los migrantes fueron provistos de recursos para satisfacción de sus necesidades físicas (entorno saludable), económicas (empleo y seguridad), culturales (arte y recreación) y políticas (derechos civiles). Es decir, el Trabajo Social se enfocó hacia la edificación de un sistema de protección social para con los migrantes (Silva, 1997).

El trabajo social asistencialista se orientó al desarrollo social, entendido como la gestión y administración estatal de los recursos y las oportunidades en las que se incluyó a los migrantes como actores participes de la dinámica nacional, sin considerar su dimensión sostenible y sustentable (Lozaya y Palomera, 1997).

La política social, referida a un sistema de reproducción de las diferencias entre estratos y sectores sociales, no excluyó a los migrantes norteamericanos, europeos o sudamericanos, pero a los centroamericanos los confinó a una función transitoria, al asumir que su objetivo laboral estaba en Estados Unidos. Es así como la política social diferenció a las clases sin pretender reducir sus niveles de pobreza y solo mantener los privilegios que, en el caso de los migrantes, se mantuvieron intactos (Zúñiga y Trejo, 1997).

De este modo, el Trabajo Social de la migración ha adoptado los conceptos acuñados por las ciencias sociales -economía, sociología y psicología-, para abordar las problemáticas inherentes a los procesos migratorios de países expulsores a economías desarrolladas. En este sentido, las categorías de "inmigración", "refugio", “aislamiento”, “desplazamiento", “repatriación”, “indocumen- 
tación" y "transmigración" han sido empleadas por los estudios del Trabajo Social para explorar, describir, explicar o comprender las relaciones entre los flujos laborales y las remesas, la migración de menores y la trata de personas (García, 2015).

La primera categoría acuñada por las ciencias económico-administrativas fue la de "inmigrante":

An individual who has lived outside of their country of birth for more than one year with a view to permanent settlement. Temporary labor migrants, guest workers, who migrate for short periods in different parts of the world and send remittances back home: e.g. immigrants in UAE who are emigrants from other middle east countries, Philippines, India. Agricultural workers from México in the USA. Skilled professionals, e.g. nurses, doctors, IT workers who are in short supply. Family reunification migrants. International adoptees. (OECD, 2010)

Una persona que ha vivido fuera de su país de nacimiento por más de un año con vistas a un asentamiento permanente. Migrantes laborales temporales, trabajadores invitados que migran por períodos cortos en diferentes partes del mundo y envían remesas a su país de origen. inmigrantes en EAU que son emigrantes de otros países del Medio Oriente, Filipinas, India. Trabajadores agrícolas de México en los Estados Unidos. Profesionales expertos, por ejemplo, enfermeras, doctores, trabajadores que escasean. Migrantes de reunificación familiar. Adoptados internacionales. (OCDE, 2010)

En el marco de las relaciones comerciales entre los Estados Unidos de América y los países considerados como "subdesarrollados" o "en vías de desarrollo", la categoría de "inmigrante" fue propuesta para denostar a aquellas personas que se introducían en la economía agroindustrial estadounidense sin documentación y habilidad laboral alguna. Incluso la geopolítica de EE. UU permitió la entrada masiva y temporal de inmigrantes para garantizar la fuerza laboral que los conflictos bélicos requirieron durante las guerras mundiales (García, 2016). 
Precisamente, durante el armamentismo, los países satélites de las potencias mantuvieron conflictos que derivaron en la salida masiva de quienes fueron considerados refugiados:

Defined by 1951 Convention, 1967 Protocol, 1969 OAU (Organization of African Unity) Convention. Governing the Specific Aspects of Refugee Problems in Africa the 1984 Cartagena Declaration. Most important document for identifying refugee status is the 1951 Convention and its 1967 Protocol. Forms the basis of international law on refugees. Article 1A(2) of the 1951 Convention defines as a refugee any person who owing to wellfounded fear of being persecuted for reasons of race, religion, nationality, membership of a particular social group or political opinion, is outside the country of his [or her] nationality and is unable, or owing to such fear, is unwilling to avail him [or her]self of the protection of that country; or who, not having a nationality and being outside the country of his [or her] former habitual residence as a result of such events, is unable or, owing to such fear, is unwilling to return to it." the 1967 Protocol: extended the definition of refugee beyond the 1951 date defined by the protocol. The 1969 OAU (Organization of African, Unity) Convention Governing the Specific Aspects of Refugee Problems in Africa: extended the definition of refugee to Africa the 1984 Cartagena Declaration: extended the definition of refugee to Central and South America. (OECD, 2010)

Definido por el Convenio de 1951, el Protocolo de 1967, el Convenio de la OUA (Organización de la Unidad Africana) de 1969. Gobernando los Aspectos Específicos de los Problemas de los Refugiados en África la Declaración de Cartagena de 1984. El documento más importante para identificar el estatuto de refugiado es la Convención de 1951 y su Protocolo de 1967. Forma la base del derecho internacional sobre refugiados. El artículo $1 \mathrm{~A}$ (2) de la Convención de 1951 define como refugiado a toda persona que, debido al temor fundado de ser perseguida por motivos de raza, religión, nacionalidad, pertenencia a un grupo social u 
opinión política en particular, se encuentre fuera del país de su propia nacionalidad y no puede, o debido a tal temor, no está dispuesto a acogerse de la protección de ese país; o que, al no tener una nacionalidad y estar fuera del país de su residencia habitual como resultado de tales eventos, no puede o, debido a ese temor, no está dispuesto a regresar a él". El Protocolo de 1967: ha ampliado la definición de refugiado más allá de la fecha de 1951 definida por el protocolo. La Convención de la OUA de 1969 (Organización de África, Unidad), que regula los aspectos específicos de los problemas de los refugiados en África, amplió la definición de refugiado a África en la Declaración de Cartagena de 1984 y extendió la definición de refugiado a América Central $y$ del Sur. (OCDE, 2010)

La venta masiva de armamento a los países satélites de las potencias generó una economía de la guerra que no solo orilló al desplazamiento forzado de personas en riesgo que migraron a otros países circunvecinos. Los grupos de personas que se opusieron a los regímenes autoritarios, armamentistas y conflictivos, fueron considerados como asilados:

Are people who have requested international protection for fear of persecution and their life, and whose claim for refugee status is still pending. They may be in a country of asylum by having fled their country of origin, by having overstayed their visa or other entry papers, or by having gained illegal entry into a country (OECD, 2010).

Son personas que han solicitado protección internacional por temor a la persecución y por su vida, y cuya solicitud de refugio aún está pendiente. Puede que se encuentren en un país de asilo huyendo de su país de origen, al haberse olvidado o perdido su visa u otros documentos de entrada, o al haber ingresado ilegalmente en un país. (OCDE, 2010)

En la medida en que las crisis bélicas y armamentistas intensificaron los conflictos, la sociedad civil generó líderes disidentes que, 
por sus ideas, recibieron asilo político, aunque la política de estos países receptores de disidentes fue la de apoyar la carrera armamentista con el argumento de una seguridad global liderada por los EE.UU. (Carreón, Hernández y García, 2015b).

En países con algún grado de desarrollo, se generó una disidencia que amenazó con colapsar al sistema armamentista, cuando menos a nivel nacional o local. Se trata de los desplazados:

Are people who have been forced to leave their homes and who have not crossed an international border. This forced migration may occur as a result of: armed conflict, situations of generalized violence, violations of human rights, natural or human-made disasters (OECD, 2010).

Son personas que se han visto obligadas a abandonar sus hogares y que no han cruzado una frontera internacional. Esta migración forzada puede ocurrir como resultado de conflicto armado, situaciones de violencia generalizada, violaciones de los derechos humanos, desastres naturales o provocados por el hombre. (OCDE, 2010)

El final de la Guerra Fría exacerbó las crisis económicas de los países periféricos al desarrollo, ya que la justificación de la seguridad nacional, la amenaza bélica o el combate a la disidencia se justificaron a partir de una inminente guerra mundial. Pero al terminar el régimen soviético la amenaza se disolvió; aunque la inversión en armas siguió incrementándose, los flujos migratorios empezaron a explicarse por motivos económicos, calidad de vida y bienestar subjetivo (García, 2017).

En síntesis, la migración es un fenómeno constante en la historia de la humanidad. Se exacerbó en la medida en que se gestó y desarrolló la economía de la guerra, la cual consiste en la producción, compra y venta de armas de países desarrollados hacia países periféricos al desarrollo. Los conflictos generados por el armamentismo propiciaron nuevas formas de migración que legitimaron la geopolítica (García, Carreón y Valdés, 2017). 
Empero, las categorías de migración han sido abordadas en el contexto latinoamericano como áreas de oportunidad para la intervención centrada en la protección social, la cual supone una estrategia integral de prevención de riesgos, con énfasis en la promoción de los derechos económicos, políticos, sociales, laborales, ocupacionales y ambientales (Gavazo, 2011).

En ese sentido, la economía de la guerra ha sido estudiada como un contexto de conflictos entre países, que tendría un correlato en las asimetrías entre países expulsores respecto de países receptores de mano de obra laboral, aun y cuando los flujos migratorios han sido explicados como redes de oportunidades y capacidades laborales, potenciadas mediante los lazos familiares (Carreón, García y Pérez, 2017).

De este modo, tanto el esquema de ruptura, travesía, estancia y retorno, como el ciclo de los flujos migratorios, adquiere, un nuevo significado ante los desastres naturales, las catástrofes ecológicas y los riesgos ambientales, indicadores del efecto del cambio climático sobre la seguridad y la salud comunitaria, con repercusión en la calidad de vida, el bienestar subjetivo y el desarrollo humano, indicados por el tipo de empleo y el tipo de salud ocupacional en torno a la prevención de accidentes y enfermedades (González e Iglesias, 2015).

Es así como el diseño de políticas sociales y laborales gira en torno a la protección como instrumento de gestión y administración de los derechos económicos y laborales, pero las diferencias entre comunidades migrantes y culturas oriundas supone una administración pública distante de las necesidades y las expectativas sociales (Roestemburg, 2013).

\section{Historia de la migración}

La migración ha transitado cuando menos por cinco periodos que corresponden a etapas de desarrollo económico. En el primer periodo, que va de 1800 a 1925, durante las revoluciones industriales, los europeos migraron hacia EE.UU., América Latina y Aus- 
tralia, influidos por la expansión de la economía (Aysa y Cachon, 2013). En la segunda etapa, que va de 1930 a 1960, la migración se ve frenada por los conflictos y guerras mundiales en Europa. En el tercer periodo, que va de 1960 a 1990, la migración adquiere un carácter global, ya que el crecimiento económico de los países asiáticos desplazó a los Estados Unidos como epicentro del desarrollo económico y tecnológico, propiciando que la mano de obra se desplazara hacia las economías llamadas "tigres asiáticos". El inició del actual ciclo condicionó las políticas restrictivas a la migración por motivos terroristas más que económicos o geopolíticos. En este sentido, se exacerbaron las medidas de seguridad en el control de los flujos migratorios. En la actual década el 54\% de los países desarrollados buscan contener los flujos migratorios; $22 \%$ con tasas a la baja y $30 \%$ siguen políticas de criminalización (Pérez, Rivero y Uribe, 2014).

La migración económica por motivos de búsqueda de oportunidades laborales predomina en el panorama histórico, pero emergen nuevos flujos migratorios que obedecen a redes de identidad y conocimiento, como son los casos de trabajadores y estudiantes (Martínez, 2014).

En este nuevo contexto económico, político, social y ambiental, los flujos migratorios parecen obedecer a demandas, recursos y oportunidades diversas, en torno a las que sus capacidades, habilidades y conocimientos los han llevado a una selección intensiva de su fuerza laboral, así como de su formación profesional (Barrón, 2013).

Se estima que el $40 \%$ de los nuevos empleos no existía hace cinco años y que el $60 \%$ de la economía se explicaría por las redes solidarias y cooperativas en las que los flujos migratorios parecen cobrar relevancia al ser instrumentos, no solo de protección social de sus derechos, sino fuente de iniciativas y diálogos interculturales en torno a los que se edifican nuevos acuerdos, tratados y leyes de representación política, jurídica y gubernamental de las minorías, con la finalidad de equilibrar las fuerzas que ejecutan o legislan la prevención de desastres, accidentes y delitos relativos a los 
desencuentros entre comunidades migrantes y culturas oriundas (García et al., 2013).

Por consiguiente, la formación profesional, la gestión del conocimiento y la producción de saberes son ejemplos del diálogo entre las comunidades migrantes respecto de los aportes oriundos en el plano de la seguridad laboral, la salud ocupacional y el bienestar subjetivo (Meer y Modood, 2011).

\section{Datos coyunturales de la migración}

Los países menos desarrollados hospedan al 81\% de la población, hasta 2005, alrededor de 191 millones de flujos migratorios de los cuales solo el 3\% proviene de los países desarrollados. En esas economías, los migrantes explican el 10\% del total de economías desarrolladas. Uno de cada cinco migrantes se estableció en EE.UU., pero el resto está en las economías asiáticas debido a la prosperidad de la región (Berry, 2016).

Los flujos migratorios reflejan la geopolítica de EE.UU. y Europa, así como la prosperidad de los países de Asia; empero, los conflictos entre países periféricos al desarrollo y el crecimiento económico, como medio Oriente, han incrementado el flujo de refugiados y asilados.

Más aún, se sabe que los desplazamientos forzados por motivos bélicos han sido superados por los desplazamientos que buscan eludir los desastres naturales, las catástrofes ecológicas y las crisis ambientales, como es el caso de las enfermedades hidrotransmitidas que matan a un millón de infantes al año (Carreón, Hernández y García, 2015).

Es decir, los flujos migratorios develan el anacronismo de las políticas, estrategias y programas de protección social y civil en materia de seguridad social, derechos humanos y formación profesional. Se trata de un nuevo panorama en el que los flujos migratorios parecen estar en el centro de la atención, al evidenciar la dimensión intercultural de las diferencias entre migrantes y oriundos (García et al., 2015). 


\section{Teorías de la migración}

Un dispositivo es un escenario de reproducción de la dominación social que necesariamente da origen a otros dispositivos de sufrimiento, reivindicación o emancipación social. En este sentido, los marcos teóricos y conceptuales explican el funcionamiento del dispositivo que, en el caso de la migración, alude a una geopolítica de crecimiento económico y flujos migratorios (García et al., 2017).

Las teorías que explican los desencuentros entre los dispositivos geopolíticos y migratorios son la teoría neoclásica, la teoría crítica, la teoría del sistema mundo, la teoría de la segmentación, la teoría del capital social y la teoría trasnacional.

La teoría neoclásica explica los flujos migratorios a partir de las oportunidades y las demandas laborales de los países expulsores con respecto a los países receptores. Sin embargo, la teoría crítica advierte que, más bien, los flujos migratorios, si bien parten de una necesidad material de subsistencia, una vez que ésta ha sido satisfecha, son el resultado de redes de categorización e identidad (Izcara y Andrade, 2016).

No obstante, la teoría crítica devela el proceso de relaciones humanas en los flujos migratorios para enaltecer la fuerza laboral y la identidad hacia un modo de producción. Es la teoría del sistema mundo la que explica con mayor exactitud la dependencia entre los países emergentes con respecto a las economías desarrolladas. Se trata de un escenario en el que los flujos de inversión determinan los flujos migratorios y sus procesos de identidad. Incluso la teoría del sistema mundo identifica a la ideología como instrumento para diseminar la creencia de que la prosperidad se genera en los países centrales y se diseminará en los países de la periferia económica (Ryabichenko y Lebedeba, 2016).

La teoría del sistema mundo advierte que los flujos económicos y los flujos migratorios son parte de una red geopolítica en la que la economía de la guerra intensificó la migración de las economías periféricas a los países desarrollados, pero soslayó la segmenta- 
ción de los mercados como resultado de la competitividad de los países de Asia (García, 2007).

Una vez que los llamados "tigres asiáticos" intensificaron su desarrollo, los demás países de la periferia se vieron obligados a acelerar su crecimiento, pero éste fue frenado por las políticas restrictivas y proteccionistas de los EE.UU., aun y cuando este propició un incremento considerable en algunos países, como Brasil o México, la industria en estos países tuvo que fragmentarse hasta un punto tal que las maquiladoras se organizaron conforme a los estándares de calidad de los países en pleno crecimiento (Roestemburg, 2013).

Por consiguiente, los flujos migratorios ya no solo obedecieron a la aculturación, sino a la selectividad de los migrantes más capaces con respecto a las exigencias de los mercados. A diferencia de la adaptación y la asimilación de una cultura dominante, los flujos migratorios empezaron a segmentarse en mano de obra calificada que, al ser seleccionada en los países desarrollados, propiciaron un nuevo estatus migratorio que explicaría los avances en las zonas de arribo de talentos (Ryabichenko y Lebedeba, 2016).

Empero, los flujos migratorios, una vez que vieron satisfechas sus necesidades, desarrollaron redes de apoyo con la finalidad de establecer puentes de conexión entre las comunidades expulsoras y las localidades receptoras de migrantes. El resultado no solo fue la unión de ambas culturas, migrante y oriunda, sino además el establecimiento de alianzas estratégicas que conformaron una nueva visión de la migración, el empleo, la salud y la educación, ya que esta migración impactó en los ámbitos de desarrollo humano (Meer y Modood, 2011).

De este modo, se generó un capital social o redes de saberes, a partir de los cuales la reproducción del conocimiento se facilitó y orientó la emergencia de nuevos flujos migrantes, mucho más selectos y capaces de ajustar sus habilidades y conocimientos a las nuevas exigencias de las organizaciones innovadoras (García et al., 2016).

No obstante que los flujos de identidad migratoria explican la sujeción de las culturas migrantes con respecto a una cultura domi- 
nante oriunda, la teoría de la transnacionalización asegura que el proceso de migración es cíclico, ya que gracias a las redes de identidades que se establecen entre las comunidades expulsoras de origen y las localidades receptoras maquiladoras, no solo las remesas indican tal identidad, sino además el retorno de los migrantes a su lugar de origen (Izcara, 2011).

Las teorías que explican los flujos migratorios resaltan la importancia de considerar el desarrollo económico de países emergentes como los asiáticos. En esa virtud, será posible explicar la segmentación de la producción y la diversificación de la mano de obra especializada. Por consiguiente, los flujos migratorios ya no solo obedecen a la aculturación, sino además anticipan escenarios de multiculturalidad en que, institucionalmente, la cultura dominante seguirá reproduciéndose en detrimento de los aportes de otras culturas migrantes.

Sin embargo, las aproximaciones teóricas económicas solo muestran las diferencias de derechos económicos y laborales entre grupos migrantes y oriundos, pero serán los marcos sociológicos, como la teoría de la privación relativa, la teoría del multiculturalismo y la teoría del interculturalismo, los que realizarán aportes en otros sentidos (Izacarra y Andrade, 2016).

Pues bien, la distinción entre los flujos migratorios y las comunidades oriundas estriba en la emergencia de sesgos económicos y sociales, atribuibles a los migrantes por parte de los oriundos respecto a las amenazas que suponen los riesgos laborales que están dispuestos a correr, reduciendo los salarios y las condiciones mínimas de salud y prevención de accidentes y enfermedades.

La teoría de la privación relativa advierte que los grupos oriundos observan con recelo a los flujos migratorios, no tanto por su condición económica, política o social, sino por su disposición laboral a aceptar los trabajos más riesgosos y con menor salario (García, 2008).

Es así como en países desarrollados con una mezcla de culturas, la iniciativa multicultural circunscribe los usos y costumbres de 
los flujos migratorios a leyes de bienestar social. Es decir, criterios mínimos de salud ocupacional en torno a los cuales los migrantes deberán asimilar y adaptarse como prerrequisitos para emplearse en condiciones desfavorables.

Sin embargo, la prevalencia de una cultura dominante respecto de los valores y las tradiciones de flujos migrantes hizo indispensable un equilibrio en la promulgación de leyes y la ejecución de las mismas. Se trata del interculturalismo que, en su esencia sociológica, alude a un diálogo entre culturas migrantes y oriundas, pero en un plano jurídico plantea la representación proporcional de las minorías en el senado, el ejecutivo y la instancia judicial, para garantizar un juicio equitativo y dirimir las asimetrías entre los grupos.

El interculturalismo social vendrá a proponer que los flujos migratorios son incluso fundamentales para el desarrollo económico, político, social y cultural de una comunidad oriunda, pero no necesariamente a través del diálogo, la controversia, los acuerdos y las corresponsabilidades (Garcia, 2016).

No obstante que las aproximaciones sociológicas explican las diferencias grupales entre migrantes y oriundos, serán los enfoques psicológicos de las representaciones sociales, la identidad social y la disposición laboral los que explicarán los riesgos ocupacionales que los migrantes están dispuestos a correr con el fin de poder garantizarse un ingreso mínimo.

Si bien los efectos del cambio climático sobre la salud pública y ocupacional son difundidos en los medios de comunicación tradicionales, como la televisión, la radio o la prensa, las diferencias entre las representaciones sociales de oriundos y migrantes se exacerban en cuanto al acceso a la información que, en el caso de los migrantes, es limitada por el idioma y la lectoescritura (García, 2015).

Se trata de un proceso en el que la información está sesgada por los noticiarios, columnas o programas, incidiendo en la búsqueda de trabajo y el desempeño para el caso de los oriundos, pero, 
en el caso de los migrantes, las representaciones sociales se gestan en la difusión de las oportunidades laborales entre los propios migrantes.

Tales diferencias representacionales definirán las asimetrías en cuanto a las identidades laborales migrantes y oriundas, las primeras con una propensión a los riesgos y las segundas con una aversión a esos riesgos.

De este modo, las asimetrías entre las identidades migrantes y oriundas generan climas laborales que definirán las tareas, apoyos e innovaciones. Eso explica las redes solidarias entre migrantes versus las redes competitivas entre los oriundos.

Sin embargo, la formación de redes colaborativas o competitivas no siempre están respaldadas por disposiciones favorables a los riesgos laborales, las condiciones del trabajo o las demandas organizacionales. La teoría de las disposiciones explicará esas diferencias y anticipará escenarios de conflictos entre ambos grupos al interior de las organizaciones.

En general, las disposiciones son indicadoras de la formación profesional de trabajadores que, por su grado de aprendizaje, compiten por un puesto laboral, aunque en el caso de los migrantes la exigencia es mayor cuando el tipo de empleo determina la calidad de su salud ocupacional (Sobrino, 2014).

Pues bien, la salud ocupacional no será el resultado de programas sociales de protección a los migrantes trabajadores, o bien producto de la promoción del autocuidado sino de una toma de decisión centrada en los riesgos como factor de empleo o autoempleo, así como determinante del bienestar personal.

Por consiguiente, desde el trabajo social con migrantes y su salud ocupacional será fundamental especificar las condiciones laborales, los riesgos en el trabajo y las amenazas que se ciñen sobre sus empleos, accidentes y enfermedades, con el fin de poder anticipar escenarios de pérdidas de extremidades, adhesión al tratamiento de enfermedades o bien el duelo de sus familiares (Wieviorka, 2009). 
Se trata de un proceso integral que se correspondería con los riesgos y las diferencias que los migrantes experimentan en sus ámbitos laborales y en relación con los privilegios de los oriundos (García, 2017).

\section{Estudios económicos, sociológicos y psicológicos de la migración}

Los estudios de la migración en el plano económico develan la prevalencia de la aculturación, adaptación, asimilación y selectividad de migrantes considerados como mano de obra con respecto a las demandas laborales del mercado, sus capacidades, habilidades y conocimientos para la inserción al mercado laboral.

Tabla 1. Estudios económicos, sociológicos y psicológicos de la migración.

\begin{tabular}{|c|c|c|c|c|}
\hline Año & Autor & Participantes & Instrumento & Hallazgo \\
\hline 1970 & Gorz & $\begin{array}{l}\text { Inmigrantes tra- } \\
\text { bajadores }\end{array}$ & $\begin{array}{l}\text { Análisis de } \\
\text { contenido y et- } \\
\text { nometodología }\end{array}$ & $\begin{array}{l}\text { Los documentos muestran un } \\
\text { sesgo o estereotipo hacia los in- } \\
\text { migrantes trabajadores al confi- } \\
\text { narlos a labores básicas. }\end{array}$ \\
\hline 1971 & Bohning & $\begin{array}{l}\text { Migrantes del } \\
\text { Reino Unido }\end{array}$ & $\begin{array}{l}\text { Análisis obser- } \\
\text { vacional y mi- } \\
\text { crohistórico }\end{array}$ & $\begin{array}{l}\text { La identidad fue reflejada en las } \\
\text { experiencias declaradas de los } \\
\text { participantes, resaltando el vín- } \\
\text { culo con EU. }\end{array}$ \\
\hline 1975 & Castells & $\begin{array}{l}\text { Inmigrantes eu- } \\
\text { ropeos }\end{array}$ & $\begin{array}{l}\text { Análisis del } \\
\text { discurso }\end{array}$ & $\begin{array}{l}\text { Los significados del lugar de re- } \\
\text { cepción o llegada están repre- } \\
\text { sentados por el sueño americano } \\
\text { y las oportunidades laborales. }\end{array}$ \\
\hline 1978 & Bach & $\begin{array}{l}\text { In m ig r a n tes } \\
\text { mexicanos }\end{array}$ & $\begin{array}{l}\text { Entrevistas a } \\
\text { profundidad }\end{array}$ & $\begin{array}{l}\text { Los significados de la migración } \\
\text { aluden a las expectativas de pro- } \\
\text { greso. }\end{array}$ \\
\hline 1979 & Freeman & $\begin{array}{l}\text { Inmigrantes dis- } \\
\text { criminados }\end{array}$ & $\begin{array}{l}\text { Análisis crítico } \\
\text { de información }\end{array}$ & $\begin{array}{l}\text { Las formas de discriminación } \\
\text { han transitado de agresiones fí- } \\
\text { sicas a desplantes o evitación de } \\
\text { contacto. }\end{array}$ \\
\hline 1984 & Castles & $\begin{array}{l}\text { Inmigrantes eu- } \\
\text { ropeos }\end{array}$ & $\begin{array}{l}\text { Análisis obser- } \\
\text { vacional }\end{array}$ & $\begin{array}{l}\text { Categorización de inmigrantes } \\
\text { en la estancia de migración. }\end{array}$ \\
\hline 1984 & Dagodag & $\begin{array}{l}\text { Ilegales mexica- } \\
\text { nos en Estados } \\
\text { Unidos }\end{array}$ & $\begin{array}{l}\text { Análisis con- } \\
\text { versacional }\end{array}$ & $\begin{array}{l}\text { El estatus migratorio adquiere } \\
\text { un sentido de vida para quienes } \\
\text { buscan un empleo. }\end{array}$ \\
\hline
\end{tabular}




\begin{tabular}{|c|c|c|c|c|}
\hline 1985 & $\begin{array}{ll}\text { Castles } & \text { y } \\
\text { Kosack }\end{array}$ & $\begin{array}{l}\text { Inmigrantes eu- } \\
\text { ropeos }\end{array}$ & $\begin{array}{l}\text { Análisis de na- } \\
\text { rrativas de ex- } \\
\text { periencias de } \\
\text { travesía }\end{array}$ & $\begin{array}{l}\text { Las construcciones discursivas } \\
\text { giraron en torno a los riesgos y } \\
\text { los desafíos como distintivo de la } \\
\text { identidad de género. }\end{array}$ \\
\hline 1987 & Cohen & $\begin{array}{l}\text { Trabajadores mi- } \\
\text { grantes }\end{array}$ & $\begin{array}{l}\text { Entrevistas y } \\
\text { observación } \\
\text { de estudios de } \\
\text { caso }\end{array}$ & $\begin{array}{l}\text { Los significados en torno a la sa- } \\
\text { lud ocupacional se relacionaron } \\
\text { con el tipo de empleo }\end{array}$ \\
\hline 1990 & Beall & $\begin{array}{l}\text { Mujeres residen- } \\
\text { tes en EU }\end{array}$ & $\begin{array}{l}\text { Narrativas y } \\
\text { conversaciones }\end{array}$ & $\begin{array}{l}\text { Las mujeres construyeron repre- } \\
\text { sentaciones de inequidad con } \\
\text { respecto a la identidad mascu- } \\
\text { lina. }\end{array}$ \\
\hline 1991 & Heer & $\begin{array}{l}\text { Indocumentados } \\
\text { mexicanos en Es- } \\
\text { tados Unidos }\end{array}$ & $\begin{array}{l}\text { Análisis con- } \\
\text { versacional }\end{array}$ & $\begin{array}{l}\text { Predominan los significados de } \\
\text { injusticia ante la creciente ola de } \\
\text { culpabilidad a latinos y mexica- } \\
\text { nos en el sistema penal. }\end{array}$ \\
\hline 1992 & Chavez & $\begin{array}{l}\text { Indocumentados } \\
\text { en Estados Uni- } \\
\text { dos }\end{array}$ & $\begin{array}{l}\text { Entrevistas a } \\
\text { profundidad }\end{array}$ & $\begin{array}{l}\text { Los microrelatos y la microhis- } \\
\text { toria de vida de los participantes } \\
\text { giran en torno a la familia. }\end{array}$ \\
\hline 1995 & Calvita & $\begin{array}{l}\text { In migrantes } \\
\text { mexicanos }\end{array}$ & $\begin{array}{l}\text { Análisis } \\
\text { microhistórico } \\
\text { conversacional }\end{array}$ & $\begin{array}{l}\text { Los significados de vivir en el ex- } \\
\text { tranjero consistieron en la asimi- } \\
\text { lación de la cultura y la añoranza } \\
\text { del lugar de origen. }\end{array}$ \\
\hline 1995 & $\begin{array}{l}\text { Chaliand } \\
\text { y Rageau }\end{array}$ & $\begin{array}{l}\text { Diásporas o gru- } \\
\text { pos de migrantes } \\
\text { en EU }\end{array}$ & $\begin{array}{l}\text { Análisis con- } \\
\text { versacional y } \\
\text { observacional }\end{array}$ & $\begin{array}{l}\text { Los estilos de vida y discursos se } \\
\text { orientan por la exclusión y la rei- } \\
\text { vindicación de la diáspora con } \\
\text { respecto a la cultura dominante. }\end{array}$ \\
\hline 2001 & $\begin{array}{l}\text { Noh y } \\
\text { Viruell- } \\
\text { Fuentes }\end{array}$ & $\begin{array}{l}\text { Residentes de To- } \\
\text { ronto, Canadá }\end{array}$ & $\begin{array}{l}\text { Análisis de dis- } \\
\text { curso }\end{array}$ & $\begin{array}{l}\text { Los microrelatos se orientaron } \\
\text { por expectativas de empleo. }\end{array}$ \\
\hline 2003 & $\begin{array}{l}\text { Castles y } \\
\text { Miller }\end{array}$ & $\begin{array}{l}\text { Inmigrantes eu- } \\
\text { ropeos }\end{array}$ & $\begin{array}{l}\text { Observación } \\
\text { participante }\end{array}$ & $\begin{array}{l}\text { Los estilos de vida fueron indi- } \\
\text { cativos de las diferencias entre la } \\
\text { cultura dominante y las culturas } \\
\text { migrantes. }\end{array}$ \\
\hline 2004 & Futo & $\begin{array}{l}\text { Ilegales mexica- } \\
\text { nos en Estados } \\
\text { Unidos }\end{array}$ & $\begin{array}{l}\text { Análisis de } \\
\text { contenido }\end{array}$ & $\begin{array}{l}\text { La documentación revisada ad- } \\
\text { vierte la emergencia de significa- } \\
\text { dos en torno al conflicto social. }\end{array}$ \\
\hline 2010 & $\begin{array}{l}\text { Solar } \\
\text { Irwin }\end{array}$ & $\begin{array}{l}\text { Migrantes, Esta- } \\
\text { dos Unidos }\end{array}$ & $\begin{array}{l}\text { Análisis con- } \\
\text { versacional }\end{array}$ & $\begin{array}{l}\text { La salud pública es representada } \\
\text { como un derecho inalcanzable. }\end{array}$ \\
\hline
\end{tabular}

Fuente: Elaboración propia 
Los estudios económicos enfatizan el equilibrio entre las demandas del mercado y las capacidades de las organizaciones contratantes de migrantes para satisfacer los requerimientos, aunque también ponen de relieve la importancia de la formación profesional, la capacitación y el adiestramiento como elementos centrales de las políticas de fomento empresarial y microfinanciamiento (Angiano, Cruz y García, 2015).

De este modo, se sabe que existen diferencias entre hombres y mujeres respecto al microcrédito, así como en cuanto al emprendimiento y el desempeño. Se trata de un panorama en el que las mujeres se insertan más rápida y exitosamente que los hombres al mercado laboral de los servicios.

No obstante, los estudios sociológicos han avanzado hacia la observación de los derechos laborales en el sentido de la incidencia de estructuras de poder en el desempeño de los migrantes. Es el caso del acoso y hostigamiento laboral, que repercute en el desempeño femenino con respecto al masculino, pero también en cuanto a seguridad y accidentes: son los hombres quienes corren más riesgos en empresas dedicadas a los servicios industriales y de seguridad (Granados y Pizarro, 2013).

En cuanto al rubro de la responsabilidad social corporativa, las diferencias entre los géneros se acortan, pero se sabe que los trabajadores migrantes con más edad tienen una propensión al futuro que se traduce en un mayor respeto a los procesos de calidad respecto al desempeño de los jóvenes.

En cuanto al efecto que tienen los riesgos en la identidad colaborativa de los grupos de migrantes es posible advertir que la solidaridad y el apoyo, emergen en un clima de relaciones autocrático más que democrático, ya que la solidaridad en este último caso se traduce en un salario con base en las competencias más que en la colaboración.

\section{Especificación de un modelo para el estudio de la migración}

La figura 1 muestra los factores que inciden en la salud ocupacional de los migrantes desde una perspectiva intercultural, la cual 
garantizaría plenamente el respeto de sus derechos económicos y laborales, pero también anticiparía escenarios de conflictos y acuerdos desde una equidad jurídica a partir de la representación de las minorías en las organizaciones productivas.

Figura 1. Especificación de un modelo para el estudio de la salud ocupacional migratoria.

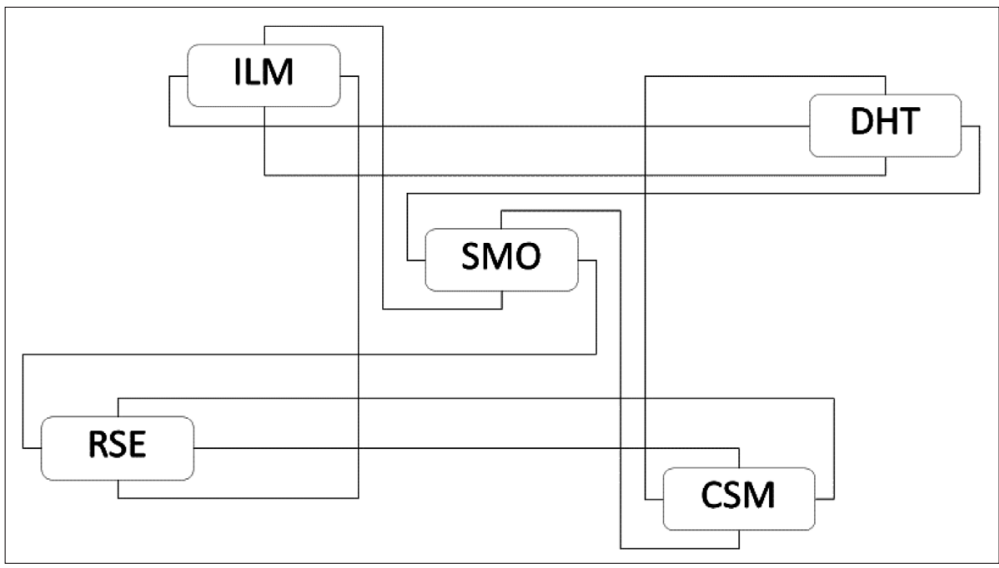

SMO = Salud Migratoria Ocupacional, DHT = Disposición hacia el Trabajo, ILM = Identidad Laboral Migratoria, RSE = Representaciones Sociales del Empleo, CSM = Capital Social Migrante

Fuente: Elaboración propia

A partir de la revisión teórica, conceptual y empírica de la historia de la migración, considerada como un efecto de la geopolítica, economía de la guerra, carrera armamentista y seguridad nacional, así como resultado del crecimiento y la prosperidad económica de países maquiladores de Asia, es posible especificar un modelo para el estudio de los flujos migratorios desde las políticas sociales.

Se puede observar que predominan dos trayectorias relativas a la explicación de los flujos multiculturales, a la vez determinados por la aculturación, la travesía y la estancia. Es el caso de los desplazamientos forzados por el cambio climático, que redujeron las oportunidades laborales de quienes viven de la economía agrícola, o bien, los flujos determinados por el clima de violencia, corrup- 
ción e impunidad en el que no solo se busca una oportunidad de trabajo, sino, además, salvaguardar la integridad. En estos flujos migratorios es posible advertir que el retorno se lleva a cabo siempre que exista una razón poderosa, como la pérdida de un familiar. Incluso, cuando es el caso, muchas veces los familiares prefieren trasladar los restos de la persona fallecida a un lugar neutral donde el migrante pueda verlo. Se trata de un proceso en el que el migrante se ajusta a los lineamientos de la cultura dominante, tanto en su lugar de origen como en el escenario de llegada.

En contraste, la hipótesis intercultural advierte que los flujos migratorios obedecen más bien a un sistema más o menos estable de expulsión, travesía, estancia, permanencia y retorno, en el que prevalece una selectividad de las capacidades, habilidades y conocimientos que el mercado local requiere para su desarrollo. A menudo se trata de grupos organizados en torno a oficios que garantizan su subsistencia, el ahorro, envío de remesas y adquisición de bienes o formación de un patrimonio. En este proceso, la identidad explica en buena medida el ciclo de la migración. Tal proceso permite la formación de una identidad migratorio-activista, que logra su máximo esplendor en la representación política de las minorías migrantes con respecto a las mayorías oriundas.

\section{Discusión}

El presente trabajo ha especificado un modelo para el estudio de los flujos migratorios a partir de la revisión de dos hipótesis concernientes al multiculturalismo e interculturalismo. En el primer caso, se trata de una imposición cultural de las leyes, usos y costumbres dominantes con respecto a grupos migrantes. En el segundo, se advierte un ciclo migratorio que inicia con la expulsión de migrantes y termina con su retorno, una vez que se ha garantizado una estructura de formación cívica y ética que permite la generación de organización de sociedad civil a favor de las minorías migrantes. Las iniciativas de las redes ciudadanas y esferas civiles permiten la propuesta de leyes que se cristalizarán en la representación de las minorías. 
Sin embargo, la historia de la migración advierte que los flujos se intensificaron luego de que la guerra fría, específicamente la geopolítica armamentista generó conflictos en países satélites de las potencias en conflicto. Por consiguiente, una buena parte de los flujos migratorios obedecieron a conflictos y en muchas ocasiones a guerras civiles o internacionales en los que se exacerbó la xenofobia y el terrorismo.

Incluso la historia de la migración advierte que hubo un periodo en el que los flujos migratorios fueron resultado del crecimiento y la prosperidad de zonas maquiladoras y corredores industriales de Asia, pero en ninguno de los casos, por motivos bélicos o industriales, se observó el cambio climático que determinaría el desplazamiento de personas por sequías, inundaciones o deslaves de comunidades.

Los efectos del cambio climático no solo representan la causa principal de futuros desplazamientos, sino además suponen la intensificación de los flujos migratorios, conforme la erosión, las sequías, las inundaciones y los deslaves se intensifiquen.

De esta manera, el estudio de la migración tiene ante sí una nueva etapa, que corresponde a la responsabilidad social de los actores económicos, políticos y sociales con respecto a la conservación de sus especies animales y vegetales que garanticen la estabilidad climática y la subsistencia de comunidades vulnerables.

En tal sentido, nuevos conceptos tales como "hipermetropía" o "indefensión" deberán incluirse en los estudios de la migración para explicar el desplazamiento de grupos humanos expuestos a las catástrofes ambientales o a los desastres naturales.

La hipermetropía es un dispositivo grupal, que consiste en reducir a su mínima expresión los efectos del cambio climático en la calidad de vida, el bienestar subjetivo o el confort residencial de quienes viven en ciudades y observan los fenómenos naturales como eventos lejanos, predecibles y controlables. O bien, aquellos individuos que miran con desdén los eventos naturales con respecto a la infraestructura citadina. 
Empero, aun cuando los fenómenos naturales no sean una amenaza real, al ser difundidos intensivamente por los medios de comunicación generan desesperanza en consumidores, ya que, si se anuncia el cambio climático como irreversible, entonces las audiencias de los medios tenderán a suponer que, ante el final de la estabilidad climática, solo queda incrementar el consumo hasta el día en que los satisfactores ya no estén disponibles.

Ambos síntomas, hipermetropía y desesperanza, explican en buena medida el comportamiento de los asentamientos humanos ante los desastres naturales, las catástrofes ambientales o los fenómenos climáticos.

\section{Conclusión}

El objetivo del presente escrito ha sido especificar un modelo para el estudio de la migración en el ámbito laboral con énfasis en la salud ocupacional, pero el tipo de selección muestral de las fuentes limita la contribución del estudio, haciéndose necesaria una revisión extensa de la literatura en repositorios como Scopus, Copernicus, Colciencias y Thomson Reuters, lo cual permitiría extender la discusión a un ámbito global. La especificación de un modelo para el estudio de la migración explica una parte de un proceso mayor que plantea los desplazamientos y flujos humanos como resultado de la geopolítica armamentista, que intensificó una economía de la guerra y la seguridad, a la vez que incentivó el cambio climático, principal causa de migración en zonas vulnerables a inundaciones, sequías o deslaves.

\section{Bibliografía}

Angiano, M., Cruz, R. y García, R. (2015). Migración internacional de retorno. Trayectoria y reinserción laboral de migrantes veracruzanos. Papeles de Población, 19, 115-147.

Aysa, M. y Cachon, L. (2013). Determinantes de la movilidad ocupacional segmentada de los inmigrantes no comunitarios en España. Revista Internacional de Sociología, 71 (2), 383413. DOI: $10.3989 /$ ris.2012.05.03 
Barrón, A. (2013). Desempleo entre los jornaleros agrícolas, un fenómeno emergente. Revista Problemas del Desarrollo, 175 (44), 55-79.

Berry, J. (2016). Special issue multiculturalism and intercultural relations: comparative analysis. Psychology in Russia, State of the Art, 9 (1), 4-23. DOI: 10.11621/pir.2016.0101

Carreón, J., García, C. y Pérez, M. I. (2017). Redes de conocimiento en torno al emprendimiento social de flujos migratorios de retorno multicultural. Sin Fin, 21 (4), 37-45.

Carreón, J., Hernández, J. y García, C. (2015a). La identidad migratoria en el establecimiento de agenda. Diálogos de Derecho y Política, 16 (7), 8-87.

Carreón, J., Hernández, J. y García, C. (2015b). Representaciones sociales del empleo en migrantes y comerciantes de Xilitla. Revista Costarricense de Trabajo Social, 27, 15-29.

Fuentes, G. y Ortiz, L. (2012). El migrante centroamericano de paso por México, una revisión a su condición social desde la perspectiva de los derechos humanos. Convergencia, 58, 157182.

García, C. (2007). La participación sostenible en la asistencia institucional en los flujos de emigrantes. Psicología Científica, 9 (29), $1-10$.

García, C. (2008). La dinámica psicosocial de las comunidades migrantes. Enfoques, 20 (2), 49-64.

García, C. (2015). Confiabilidad y validez de un instrumento que mide el desarrollo local: vulnerabilidad hídrica, precariedad laboral, intensidad migratoria e identidad resiliente. Margen, 78, 1-11.

García, C. (2016). Los estudios psicológicos de la migración: Especificación de un modelo a partir de correlaciones entre factores sociales y organizacionales. III Foro Latinoamericano "Igualdad y Desigualdad Social en América Latina: Generando Debates en Trabajo Social en Relación con otras Ciencias del Campo Social". Buenos Aires, 24 de agosto de 2013.García, C. (2017). Modelo de salud ocupacional migratoria intercultural. Ciencia y Humanismo en la Salud, $4(2), 24-31$.

García, C. Carreón, J. y Valdés, J. (2017). Límites de los modelos de la salud ocupacional: Estudio de la adhesión al tratamiento del asma en trabajadores adultos mayores migrantes del estado de México. Visión Gerencial, 16 (1), 103-118.García, C., Carreón, J., Hernández, J., Aguilar, J. A. y Rosas, F. J. (2015). Modelo de los determinantes psicosociales de 
la identidad laboral y la vivienda en ex migrantes de Xilitla, San Luis Potosí, México. Revista Internacional de Investigación en Ciencias Sociales, 3 (1), 55-69. García, C., Montero, M. E., Bustos, J. M., Carreón, J. y Hernández, J. (2013). La inseguridad migratoria en los medios impresos de la Ciudad de México. Reflexiones, 92 (1), 156-173.

Gavazzo, N. (2011). Acciones y reacciones: Formas de discriminación hacia los migrantes bolivianos en Buenos Aires. Revista de Ciencias Sociales, 24, 50-83.

González, M. e Iglesias, C. (2015). Decisiones de tenencia de vivienda y aculturación de la población extranjera residente en España. El Trimestre Económico, 82 (325), 183-209.

Granados, J. y Pizarro, K. (2013). Paso del norte, que lejos te vas quedando. Implicaciones de los migrantes de retorno en México. Estudios Demográficos y Urbanos, 28 (83), 469-496.

Izcara, S. y Andrade, K. (2016). Los traficantes de migrantes tamaulipecos empleados por empresarios agrarios estadounidenses. Noesis, 25 (50), 174-197. DOI: 10.20983/noesis.2016.2.7

Izcara, S. (2011). Redes migratorias versus demanda laboral: Los elementos que moldean los procesos migratorios. Convergencia, 57, 39-59.

Lozaya, M. y Palomera, A. (1997). Desarrollo social. En M. Sánchez (coord.), Manual de Trabajo Social (pp. 63-96). México: UNAM-ENTS.

Martínez, G. (2014). Chiapas: Cambio social, migración y curso de vida. Revista Mexicana de Sociología, 76 (3), 347-382.

Meer, N. y Modood, T. (2011). How does interculturalism contrast with Interculturalism? Journal Intercultural Studies, 33 (2), 175196. DOI: $10.1080 / 07256868.2011 .618266$

Organization for Economic Cooperation Development (2010). Statistic for countries. New York: OECD.

Pérez, M., Rivero, M. y Uribe, I. (2014). La migración desde la mirada de los empleadores de una agroindustria de los altos de Jalisco, México. Estudios Sociales, 43, 112-136.

Roestemburg, W. (2013). A social work practice perspective on migration. Maatskaplike Werk, 49 (1), 1-21. DOI: 10.15270/49$1-72$

Ryabichenko, T. y Lebedeba, N. (2016). Assimilation or integration. Similarities and differences between acculturation attitudes of migration from central Asia and Russians in central Russia. Psychology in Russia, State of Art, 9 (1), 98-111. DOI:10.11621/pir.2016.0107 
Silva, M. (1997). Necesidades sociales. En M. Sánchez (coord.), Manual de Trabajo Social (pp. 41-62). México: UNAM-ENTS.

Sobrino, J. (2014). Migración interna y tamaño de la localidad en México: Estudios Demográficos y Urbanos, 28 (87), 443-479.

Valero, A. (1997). Apuntes sobre la historia del Trabajo Social en México. En M. Sánchez (coord.), Manual de Trabajo Social (pp. 9-40). México: UNAM-ENTS.

Wieviorka, M. (2009). Identidades, globalización e inequidad. Puebla: UIA.

Zúñiga, E. y Trajo, M. (1997). Política social. En M. Sánchez (coord.), Manual de Trabajo Social (pp. 97-117). México: UNAMENTS. 